\title{
Lingua e potere in Pier Paolo Pasolini
}

\author{
Francesco Virga \\ Palermo \\ npvirga@libero.it
}

La lingua è la spia dello spirito. ${ }^{1}$

I potenti $[\ldots]$ che in questi anni hanno detenuto il potere, dovrebbero andarsene, sparire [...]. Invece non solo restano al potere, ma parlano. Ora è la loro lingua la pietra dello scandalo. [...] La loro lingua è la lingua della menzogna. ${ }^{2}$

$\grave{\mathrm{E}}$ attraverso lo spirito della televisione che si manifesta in concreto lo spirito del nuovo potere. ${ }^{3}$

\begin{abstract}
L'analisi delle diverse forme del linguaggio umano e dei suoi poteri, la questione dei rapporti tra lingua e dialetti, con i suoi nessi stretti con la politica e la società, rappresentano una delle preoccupazioni centrali e insieme una costante dell'opera pasoliniana.

Pasolini non venne mai meno al dovere per lui "primo» di un intellettuale: «esercitare prima di tutto e senza cedimenti di nessun genere un esame critico dei fatti». Fin da giovane concepì tale esame come una verifica continua, un continuo adattamento del periscopio all'orizzonte dei fenomeni, contro gli ideologi d'ogni tipo che hanno sempre fatto il contrario. Fu così tra i primi ad intravedere gli albori di una nuova epoca storica che -oltre a cancellare i tratti dell'antica civiltà contadina, ancora propria di gran parte dell'Italia nei primi anni '60 — avrebbe finito per mutare «antropologicamente» gli italiani.

L'articolo intende dimostrarlo, passando in rassegna tutti gli scritti dell'autore, non solo quelli linguistici, e soffermandosi su alcuni testi meno noti e poco studiati o trascurati dalla critica.
\end{abstract}

Parole chiave: lingua-potere; lingua-dialetti; Gramsci; televisione; consumismo; mutazione antropologica.

1. P. P. PASOlini, Saggi sulla letteratura e sull'arte (d'ora in poi SLA), II, Milano: Mondadori, 1999 , p. 2844.

2. Trattatello pedagogico incompiuto, intitolato Gennariello, uscito a puntate su Il Mondo (primavera 1975), poi in P. P. PASOLINI, Lettere luterane, Torino: Einaudi, 1976, p. 29.

3. P. P. PASOlini, Scritti corsari, Milano: Garzanti, 1975, p. 30. 


\section{Abstract}

Analysing different forms of human language and its powers, the issue of language-dialect connections, strictly intertwined with politics and society, represent one of the author's main concerns and, at the same time, a constant feature of Pasolini's work.

Pasolini never compromised on what he believed to be his "primary" duty as an intellectual: «To perform, first and foremost, a critical examination of the facts». Since youth, he conceived of this examination as a continuous assessment, continuous focussing of his periscope to the horizon of phenomena, opposing ideologues of every kind who always did the opposite. He was therefore amongst the first to glimpse the dawn of a new historical era that - in addition to eliminating the traits of an ancient agricultural civilization, which still defined most of Italy in the early '60s — would eventually «anthropologically» transform Italians.

This article aims to demonstrate the above by reviewing all of the author's works, not only his linguistic ones, and to focus on some pieces that are lesser-known, rarely studied or neglected by critics.

Keywords: language-power; language-dialects; Gramsci; television; consumerism; anthropological transformation.

Nell'ampia bibliografia critica esistente su Pasolini gli studi che hanno messo a fuoco le sue riflessioni sui rapporti tra lingua e potere sono pochi; ${ }^{4}$ eppure, l'analisi delle diverse forme del linguaggio umano e dei suoi poteri, la questione dei rapporti tra lingua e dialetti, con i suoi nessi stretti con la politica e la società, come rilevato per primo da Tullio De Mauro, ${ }^{5}$ occupano un posto centrale nell'opera del poeta e attraversano tutti i suoi scritti. Il Volgar'eloquio, ${ }^{6}$

4. Esiste solo una bella monografia —rielaborazione della tesi di laurea - di Francesco FERRI, Linguaggio, passione e ideologia. Pier Paolo Pasolini tra Gramsci, Gadda e Contini, Roma: Progetti Museali Editore, 1996. Non a caso lo studio fu seguito e incoraggiato da Tullio DE MAuro, autore dei brevi ma fondamentali: Pasolini critico dei linguaggi e Pasolini linguista, scritti in tempi diversi e raccolti nel suo L'Italia delle Italie, Roma: Editori Riuniti, $1992^{2}$. Va inoltre ricordato: Gustavo BURATTI, «Pasolini: dialetto rivoluzionario e minoranze linguistiche», l'impegno, XIV 3, dicembre 1994. Si devono, infine, ad Antonio PIrOMALLI prefazione e cura degli Atti del dibattito, svoltosi al Liceo Palmieri di Lecce (21 ottobre 1975) con la partecipazione attiva di Pasolini: Volgar'eloquio, Napoli: Athena, 1976. Se non s'è visto male, non risultano altri contributi a stampa che, fin dal titolo, affrontano espressamente la tematica linguistica in Pasolini. Comunque, per la centralità da essa rivestita nella sua opera complessiva, è corso l'obbligo di consultare tutti i principali studi pasoliniani.

5. T. De Mauro, L'Italia delle Italie cit., p. 274: «Grazie a Gramsci, Pasolini intese che le scelte di linguaggio non avevano solo premesse e conseguenze di natura letteraria, ma si inscrivevano nel tessuto dei rapporti e contrasti sociali, avevano [...] una valenza politica [...]. Questa interpretazione di Gramsci non solo fu significativa per orientare il seguito dell'attività intellettuale di Pasolini, ma per la sua interna originalità. Essa scorgeva con chiarezza la natura portante che le questioni di lingua e, dunque, di cultura avevano nella teoria politica e sociale di Gramsci, ciò che solo molto lentamente, più di vent'anni dopo si doveva acquisire».

6. Titolo dantesco scelto da Pasolini per l'intervento testè citato (ora SLA, p. 2825-2862). Gli scritti pasoliniani, pubblicati nel tempo da editori diversi, sono stati raccolti in dieci 
oltre ad essere il tema del suo ultimo intervento pubblico, è stato al centro dei suoi interessi fin dagli anni giovanili, quando era «un linguista ossessionato».?

Indubbiamente con gli anni Pasolini ha affinato i suoi strumenti d'analisi e si scorgono facilmente le differenze esistenti tra l'articolo Dialet, lenga e stil, pubblicato nel 1944 su una rivistina provinciale friulana, e i saggi e gli articoli dei decenni successivi.

D'altra parte chi, fin dagli anni ' 50 , aveva concepito l'attività critica come una verifica continua, un continuo adattamento del periscopio all'orizzonte dei fenomen $i^{8}$ contro gli ideologi d'ogni tipo che hanno sempre fatto il contrario, era particolarmente predisposto a cogliere e decifrare i segni dei tempi. Così Pasolini è stato tra i primi ad intravedere gli albori di una nuova epoca storica che, oltre a cancellare i tratti dell'antica civiltà contadina, caratterizzante gran parte dell'Italia ancora nei primi anni ' 60 , avrebbe finito per mutare "antropologicamente» gli stessi italiani.?

La valutazione critica della complessa opera pasoliniana, è ancora oggi controversa. Il carattere non sistematico degli scritti, il loro continuo sforare i tradizionali confini disciplinari, si prestano a letture ed interpretazioni diverse. Nessuno può avere la pretesa di possedere l'unica chiave interpretativa giusta. Ogni interpretazione deve essere consapevole della propria parzialità ed essere offerta alla discussione come ipotesi di lavoro.

Tutti hanno riconosciuto il legame stretto esistente tra vita e opera in Pasolini, tanto che ad alcuni egli è apparso come la "dimostrazione vissuta», un «martire», in senso etimologico, dei propri pensieri. ${ }^{10}$ Ma non tutti hanno colto la continuità di fondo della sua opera. Chi scrive ritiene che sia, quanto meno, discutibile asserire che, a partire dagli anni '70, si delinei un «nuovo Pasolini». ${ }^{11}$ È stato, peraltro, lo stesso poeta a segnalare questa continuità, sia nella famosa lettera aperta indirizzata ad Italo Calvino nel luglio del 1974,

volumi, a cura di Walter SitI e Silvia De Laude («I Meridiani», Milano: Mondadori). La sistemazione non ha ottenuto consensi unanimi. Particolarmente dure le osservazioni di Carla BenEDETTi (L'Unità, 29 aprile 2003), che prende le mosse dalla discutibile Postfazione con cui Siti chiude l'ultimo tomo dell'opera. Soprattutto contestabile, per me, la separazione dei saggi letterari da quelli socio-politici ed antropologici (Saggi sulla politica e la società, d'ora in poi SPS). Forse, rispettando l'ordine cronologico in cui furono concepiti tutti gli scritti, a prescindere dal loro genere, sarebbe stato più agevole cogliere il ritmo e il naturale sviluppo del pensiero di un autore che non amava i confini disciplinari.

7. P. P. PASOLINI, I parlanti (1951), ora in Appendice a Ragazzi di vita, Torino: Einaudi, 1979, p. 230.

8. P. P. Pasolini, Passione e ideologia, Milano: Garzanti, 1960, p. 486-487.

9. Per tutti, P. P. PASOLINI, «Studio sulla rivoluzione antropologica in Italia», in Scritti corsari, cit. È singolare che l'intuitivo giudizio sul particolare sviluppo del capitalismo italiano abbia ora ricevuto conferme da parte di un economista: Giulio SAPELLI, Modernizzazione senza sviluppo. Il capitalismo secondo Pasolini, Milano: Bruno Mondadori, 2005, il quale ha valorizzato sapientemente "lo sguardo antropologico» del poeta, ma ha preso anche qualche abbaglio, come quando tenta di far passare Gramsci per «un attualista gentiliano» (ibid., p. 27).

10. Da ultimo Roberto CARnERo, Morire per le idee. Vita letteraria di Pier Paolo Pasolini. Con un'appendice sul caso giudiziario, Milano: Bompiani, 2010, p. 7-9.

11. Cesare SEGRE, Vitalità, passione, ideologia. Introduzione a SLA, I, p. XXXVI-XXXVII. 
esaminata più avanti, sia nella Nota introduttiva agli Scritti corsari, inspiegabilmente trascurata dalla critica, ${ }^{12}$ i cui passaggi chiave si ripropongono:

La ricostruzione di questo libro è affidata al lettore. È lui che deve rimettere insieme i frammenti di un'opera dispersa e incompleta. È lui che deve ricongiungere passi lontani che però si integrano. È lui che deve organizzare $i$ momenti contraddittori ricercandone la sostanziale unitarietà. È lui che deve eliminare le eventuali incoerenze (ossia ricerche o ipotesi abbandonate). [...] Mai mi è capitato nei miei libri, più che in questo di scritti giornalistici, di pretendere dal lettore un così necessario fervore filologico. Il fervore meno diffuso del momento. Naturalmente, il lettore è rimandato [...] ai testi degli interlocutori con cui polemizzo o a cui con tanta ostinazione replico o rispondo. Inoltre, all'opera che il lettore deve ricostruire, mancano del tutto dei materiali [...] fondamentali. $\mathrm{Mi}$ riferisco soprattutto a un gruppo di poesie italo-friulane [...]. Non potevo raccogliere qui quei versi, che non sono "corsari» (o lo sono molto di piü). ${ }^{13}$

Le poesie, cui si fa cenno, non sono quelle famose del 1941-42, ma un gruppo di versi, meno noti, scritti tra il 1973 e il 1974, che si richiamano ai precedenti. Nell' «Autopresentazione» di tutti i versi friulani, editi nel 1975, con il titolo La nuova gioventù, lo stesso Pasolini rivendica ironicamente il diritto a diventare "un po'diverso» in trent'anni e ammette di sentirsi ossessionato dal pensiero di «non aver detto e non poter dire la parola ultima e definitiva, o almeno precisa, sull'unica cosa che gli sta a cuore». ${ }^{14}$ L'affermazione risulta di per sé enigmatica. Ma Pasolini non sempre è stato chiaro, anzi ha fortemente subito il fascino dell'enigma e del mistero. D'altra parte era convinto che «bisogna essere folli per essere chiari» e che "alcune cose si vivono soltanto; o, se si dicono, si dicono in poesia». ${ }^{15}$ In ogni caso, secondo me, non

12. Con l'eccezione di Marco Belpoliti, «Pasolini corsaro e luterano», Nuovi Argomenti, 21 (2003), che ad essa fa espresso riferimento, ricordando la singolare revisione pasoliniana del 1974 dei primi versi friulani, la cui analisi è però rinviata ad altri tempi e luoghi. Ciò che non convince, nella pur originale rilettura di Belpoliti —ripresa nel suo recente Pasolini in salsa piccante, Parma: Guanda, 2010 — è la presunta centralità della tematica omosessuale, di cui certo non si può ignorare la presenza, ma che credo riduttivo considerare nodale, se non ossessiva. Non è, inoltre, confermato dall'analisi testuale che, negli scritti degli anni ' 70 , l'analisi linguistica è sostituita da quella semiologica. Basti ricordare che i principali saggi semiologici sono certo raccolti in Empirismo eretico, Milano: Garzanti, 1972, ma erano stati pubblicati in diverse riviste tutti negli anni '60. Peraltro Pasolini, proprio per il suo tipico "empirismo eretico», non fu mai seguace ortodosso di alcuna scuola di pensiero. Per questo, fin dagli anni ‘60, riesce a conciliare Gramsci e Contini, e il suo marxismo eterodosso con la psicoanalisi e lo strutturalismo. Non a caso Contini apparirà ai suoi occhi fino all'ultimo, «il solo critico italiano i cui problemi siano stati i problemi letterari di Gramsci [...] scandalo per $i$ Giudei, stoltezza per $i$ Gentili». Cfr. P. P. PASOlInI, Descrizioni di descrizioni, Torino: Einaudi, 1979, p. 443.

13. Scritti corsari, cit., p. 1-2. Salvo diverso avviso, il corsivo dei testi citati è mio.

14. P. P. PASOlInI, La nuova gioventù. Poesie friulane 1941-1974, Torino: Einaudi, 1975, p. 267.

15. P. P. PAsolini, Il sogno del centauro, ed. Jean Duflot, Roma: Editori Riuniti, $1993^{2}$ (d'ora in poi DufLot), p. 5. Ė una delle più importanti interviste rilasciate da Pasolini a cavallo degli anni ' $60-{ }^{\prime} 70$. 
ha mai smesso di svolgere quello che riteneva il «primo dovere di un intellettuale», ossia "esercitare prima di tutto e senza cedimenti di nessun genere un esame critico dei fatti». ${ }^{16}$

Non pare casuale, poi, che nell'articolo Che cos'è questo golpe? (Corriere della sera, 14 novembre 1974), Pasolini segua lo stesso metodo ed eserciti proprio quel fervore filologico, sopra invocato, nel tentativo di spiegare alcuni dei miste$r i$ italiani:

Io so perché sono un intellettuale, uno scrittore che cerca di seguire tutto ciò che succede, di conoscere tutto ciò che non si sa o che si tace; che coordina fatti anche lontani, che mette insieme i pezzi disorganizzati e frammentari di un intero coerente quadro politico, che ristabilisce la logica là dove sembrano regnare l'arbitrarietà, la follia e il mistero. ${ }^{17}$

Nelle pagine che seguono, anticipando i risultati di uno studio più analitico in corso, si mostrerà come il poeta sia riuscito a rompere il circolo perverso che lega il potere della lingua alla lingua del potere, e abbia saputo trovare parole chiare per svelare «le menzogne che attraverso la stampa e soprattutto la televisione inondano e soffocano quel corpo del resto inerte che è l'Italia». ${ }^{18}$ Si cercherà, inoltre, di applicare il metodo pasoliniano per «rimettere insieme i frammenti di un'opera dispersa e incompleta», "ricongiungere passi lontani che però si integrano", "ristabilire la logica là dove sembrano regnare l'arbitrarietà, la follia e il mistero", facendo particolare riferimento agli articoli apparsi su giornali e periodici diversi, nell'arco di trent'anni, evidenziandone la sostanziale coerenza, ma senza «sollecitare i testi ${ }^{19}$ o chiudere gli occhi di fronte alle contraddizioni del pensiero dello scrittore che, comunque, risultano sempre feconde. ${ }^{20}$

\section{Il Gramsci di Pasolini}

Pasolini ha dichiarato di aver letto Gramsci, per la prima volta, nel 1948-49, anni in cui cominciano a vedere la luce, seppure in modo incompleto, $i$ suoi scritti. ${ }^{21} \mathrm{Ma}$ l'assimilazione critica del pensiero del sardo è successiva a questo

16. Scritti corsari, cit., p. 31 .

17. Ora col titolo «Il romanzo delle stragi», ibid., p. 108.

18. Lettere luterane, cit., p. 29.

19. Espressione di Antonio Gramsci, che tanto contribuí alla formazione di Pasolini. Sarebbe tradire entrambi dimenticarsi del giudizio severo del sardo contro la diffusa tendenza a «far dire ai testi, per amor di tesi, più di quanto i testi realmente dicono», un "errore di metodo filologico» che «si verifica anche all'infuori della filologia, in tutte le analisi e gli esami delle manifestazioni di vita» (A. GramscI, Quaderni del carcere, ed. V. GerRatana, II, Torino: Einaudi, 1975, p. 838).

20. Tra i tanti critici che hanno evidenziato la "contraddizione costitutiva" dell'opera pasoliniana: Vittorio SpINAZZOLA, La modernità letteraria, Milano: Il Saggiatore, 2001, ripreso da R. CARnero, Morire per le idee, cit., p. 9.

21. L'opera di Gramsci —è noto - vide la luce, da Einaudi, con la supervisione di Palmiro Togliatti. Uscirono dapprima le Lettere dal carcere (1947), poi i volumi tratti dai Quaderni 
biennio. Ciò è dimostrato, tra l'altro, da un testo inedito del marzo $1949,{ }^{22}$ che affronta il tema classico dei rapporti tra cultura e politica, senza alcun riferimento al lessico e alla filosofia gramsciana, e da una lettera a Carlo Betocchi dell'ottobre 1954, dove l'autore svela con candore le proprie incertezze ideologiche. ${ }^{23} \mathrm{E}$ probabile che, in un primo momento, Pasolini sia rimasto colpito dalla statura morale dell'uomo imprigionato dal regime fascista: «tanto più libero — scriverà- quanto più segregato dal mondo, fuori del mondo, in una situazione suo malgrado leopardiana, ridotto a puro ed eroico pensiero». ${ }^{24}$

Solo dai primi anni '60 è possibile trovare, negli scritti di Pasolini, tracce significative del pensiero gramsciano. Soprattutto se si considera l'aspetto più problematico di esso, ch'egli è stato tra i primi a cogliere. Occorre, infatti, tenere sempre presente che il Gramsci di Pasolini non è quello ufficiale del PCI, ma il Gramsci studioso del linguaggio e del senso comune che riesce a vedere, anche nelle più elementari espressioni umane, una particolare visione del mondo. ${ }^{25}$

Da questo punto di vista, l'intera rubrica curata dal 1960 al 1965 su Vie Nuove, settimanale popolare comunista diretto da Maria Antonietta Macciocchi, ha un'inconfondibile impronta gramsciana. Non è un caso che, intrattenendovi uno straordinario dialogo con lettori spesso incolti, a proposito di questioni linguistiche, Pasolini elogi il glottologo Graziadio Isaia Ascoli e, una volta asserito che "Gramsci non è mai normativo", citi a memoria un passo dei Quaderni: "Ogni volta che affiora, in un modo o nell'altro, la questione della lingua, significa che si sta imponendo una serie di altri problemi». ${ }^{26}$

Quello praticato da Pasolini in Vie Nuove è un giornalismo rivoluzionario, assai vicino al modello gramsciano. ${ }^{27}$ Forse, persino superiore allo stile corsaro

del carcere, tematicamente cosí raccolti: Il materialismo storico e la filosofia di Benedetto Croce (1948), Gli intellettuali e l'organizzazione della cultura (1949), Il Risorgimento (1949), Note sul Machiavelli, sulla politica e sullo Stato moderno (1949), Letteratura e vita nazionale (1950), Passato e presente (1951). In più volumi saranno infine riuniti gli scritti giornalistici del periodo pre-carcerario. Bisogna aspettare il 1975 per avere, dei Quaderni, un'edizione critica (sup., n. 19).

22. Il testo, preparato per il Congresso provinciale del PCI di Pordenone, appare, per la prima volta, su Rinascita, 4 novembre 1977; ora SPS, p. 81-84.

23. P. P. Pasolini, Vita attraverso le lettere (ed. G. NAldini), Torino: Einaudi, 1994, p. 163165.

24. Passione e ideologia, cit., p. 487. L'accostamento a Leopardi torna in un'intervista del 1963, dove afferma: «l'unico antenato spirituale che conta è Marx e il suo dolce, irto, leopardiano figlio, Gramsci» (A. Arbasino, Sessanta posizioni, Milano: Feltrinelli, 1971; ora SPS, p. 1573). Nella stessa intervista Pasolini, ricordati anche i debiti contratti con Longhi e Contini, assicura di non averli dimenticati, «perché la mia caratteristica principe è la fedeltà». Non manca però di dare una stoccata a quanti «hanno fatto di tutto per rendere intollerabile l'uso dei padri» (ibid., p. 1573-1574).

25. T. De Mauro, Prefazione a P. P. Pasolini, Le belle bandiere [1977], Roma: Editori Riuniti, 1991, p. 8, ha osservato che il PCI «non ha mai saputo attuare la lezione di Gramsci».

26. Ibid., p. 86-88 e 229.

27. Nel lessico pasoliniano di quegli anni ricorrono espressioni gramsciane. Così, ad es., il brano: «So quanto l'operazione giornalistica sia falsa: prende, della realtà, brani isolati, appariscenti [...]. Non pensa il giornalista borghese, nemmeno per un istante, a servire la verità» (Le belle 
degli anni '70 che rimane legato al modello tradizionale dell'intellettuale vate o profeta, dei cui limiti Pasolini mostra di essere ben conscio fin dal 1962. Infatti, ad un lettore che gli chiede se un grande scrittore, denunciando le ingiustizie, possa mettere in crisi la società, replica:

No, non credo possibile che l'opera di uno solo, per quanto grande, possa porre in crisi uno stato di cose. [...] La democrazia ha abituato, bene o male, alla discussione e alla partecipazione: lo scrittore-oracolo non è concepibile in tempi di produzione in serie. $\dot{E}$ una figura del passato (se mai ce ne sono stati): tipica di una civiltà agricolo-artigianale. Ma anche ammesso che ci fosse uno scrittore di tale levatura [...] da poter mettere in crisi una società, egli verrebbe inesorabilmente sconfitto, oggi, dalla potenza industriale, dalle catene dei giornali e dei mezzi di diffusione conservatori e reazionari. ${ }^{28}$

Un critico severo ed esigente, come Franco Fortini, ha individuato proprio nelle pagine di Vie Nuove il Pasolini migliore. ${ }^{29}$ Lo stesso scrittore, nel dicembre 1962, poco prima di partire per quel viaggio in Africa che lascerà un segno indelebile nella sua vita, ${ }^{30}$ nel congedarsi dai lettori della sua rubrica, la definirà «uno dei punti fermi di questi ultimi anni: addirittura, in certi momenti neri, un'ancora di salvezza». ${ }^{31}$

Rileggendo queste pagine - scritte, peraltro, in una delle fasi più convulse e creative dell'autore che aveva trovato nel cinema una nuova via per esprimersi- colpisce l'eccezionale capacità di ascolto insieme alla volontà di capire e di essere capito. Nei dialoghi coi lettori Pasolini parlerà di tutto; ma due costanti, fra loro congiunte, predomineranno: la riflessione sui rapporti tra comunismo e cristianesimo e l'analisi dei rapporti tra le diverse forme del linguaggio umano e le cose.

Va precisato, però, che il Pasolini di quegli anni è cosí lontano dalla vulgata marxista-leninista, allora in voga, da essere ritenuto eretico, non solo dai lettori

bandiere, cit., p. 53), riecheggia in modo impressionante una lettera (12 ottobre 1931) di Gramsci: «Io non sono mai stato un giornalista professionista, che vende la sua penna a chi gliela paga meglio e deve continuamente mentire perché la menzogna entra nella sua qualifica professionale. Sono stato giornalista liberissimo, sempre di una sola opinione, e non ho mai dovuto nascondere le mie convinzioni per fare piacere a dei padroni o manutengoli» (A. GramSCI - T. SCHUCHT, Lettere 1926-1935, Torino: Einaudi, 1997, p. 833-834).

28. P. P. PASOlini, "Il cinema e la poesia», Vie Nuove, 6 dicembre 1962, ora Le belle bandiere, cit., p. 209-210.

29. F. Fortini, Pasolini e le ultime illusioni (1977), ora Attraverso Pasolini, Torino: Einaudi, 1993. Quanto Pasolini tenesse al giudizio di Fortini è stato evidenziato da Enzo Golino, Tra lucciole e Palazzo. Il mito Pasolini dentro la realtà, Palermo: Sellerio, 1995, p. 91-113.

30. È stato giustamente notato che l'Africa, negli anni '60, prende il posto del Friuli nell'immaginario pasoliniano: «Dal Friuli alle borgate romane, al meridione d'Italia, all'Africa, all'India si susseguono in Pasolini le tappe di un'interrotta ricerca di nuove incarnazioni del mito di un'umanità vergine e primitiva: sempre più a sud, sempre più lontano dall'odiata civiltà neocapitalistica e borghese, verso mondi ancora barbari e incontaminati». G. SANTATO, «L'abisso tra corpo e storia. Pasolini fra mito, storia e dopostoria», Studi pasoliniani, $1,2007$.

31. Le belle bandiere, cit., p. 210-211. 
più sprovveduti, ma persino dagli intellettuali marxisti più aperti. ${ }^{32} \mathrm{~A}$ tutti replica, con grande mitezza ed efficacia, lasciandoci pagine esemplari. Così, a chi gli chiede di rompere con il proprio passato piccolo-borghese, risponde:

La sua è una richiesta mistica. Lei pensa l'ideologia come un'ascesi. Questi 'distacchi' dal proprio io, dal proprio passato (che è poi la storia), sono tipici delle conversioni 'nevrotiche' che hanno caratterizzato tante santità. I marxisti non sono dei santi: sono degli uomini. La loro vita, la loro opera, la loro lotta si svolge nella storia: e la storia è una mescolanza inscindibile di passato, presente e futuro. ${ }^{33}$

Lo stesso concetto sarà ripreso nell'articolo Cultura contro nevrosi (3 maggio 1962):

essere marxisti, oggi, in un paese borghese, significa essere ancora in parte borghesi. Finché i marxisti non si renderanno conto di questo, non potranno mai essere del tutto sinceri con se stessi. La loro infanzia, la loro formazione, le loro condizioni di vita, i loro rapporti con la società, sono ancora oggettivamente borghesi. La loro 'esistenza' è borghese, anche se la loro coscienza è marxista. ${ }^{34}$

Pasolini è sempre più convinto che il marxismo non va chiuso in un sistema fisso, altrimenti diventa la copia capovolta del dogmatismo clericale. Soprattutto significativa pare cosí la replica a Lucio Lombardo Radice che, nel luglio 1962, gli attribuisce le sommarie equazioni Cristo = Marx e DC = fascismo:

Io non scrivo solo questa rubrica "parlata» [...] Mi integri con gli altri miei scritti, e non mi faccia dire quello che non voglio dire! Non ho mai inteso inglobare Gesù in Marx! [...] Ho sostenuto [...] che nulla di ciò che è stato sperimentato storicamente dall'uomo, può andare perduto: e che quindi non possono andare perdute neanche le parole di Cristo. Esse sono in noi, nostra storia. E io sono ancora (e ancora ingenuamente) convinto che per un borghese una buona lettura del Vangelo è sempre un fertilizzante per una buona prassi marxista.

Quanto alla "DC come nuovo fascismo", io ho solo citato il mio corrispondente con una certa simpatizzante ironia. Non volevo dire che la DC è, alla lettera, un nuovo fascismo. Le faccio notare, ad ogni modo, che la borghesia italiana che ha espresso il fascismo è la stessa che esprime la DC: la sfido a elencare sostanziali differenze nel campo della scuola, della magistratura, della

32. Intervenendo nel dibattito sui rapporti marxismo-cristianesimo in corso su Vie Nuove, un intellettuale non codino come Lombardo Radice lo accuserà di eresia e dilettantismo. Cfr. ibid., p. 179-183. Critiche analoghe gli rivolgeranno, in seguito, Asor Rosa, Salinari, Sanguineti, Calvino ed altri. Questi ed altri critici saranno più tardi bollati come «nuovi chierici».

33. P. P. Pasolini, «Mistica e storia», Vie Nuove, 27 maggio 1961, ora Le belle bandiere, cit., p. 102.

34. Ibid., p. 171. 
polizia, della pubblica amministrazione, dei rapporti con la potenza clericale del Vaticano. E la sfido a dimostrarmi anche le ragioni vere, culturali nel senso gramsciano della parola, per cui la DC può essere definita, come lei fa, un "grande partito cattolico». Quale cultura ha mai espresso? 35

Sul valore rivoluzionario del Cristianesimo Pasolini tornerà più volte, soprattutto, nel 1964, dopo Il Vangelo secondo Matteo, dedicato alla memoria di Giovanni XXIII. Il film, nonostante i riconoscimenti della critica, sarà accolto con freddezza e diffidenza dai settori più retrivi del mondo cattolico e comunista. E il poeta dovrà dare spiegazioni anche su Vie nuove:

Non sono affatto cattolico, anzi sono certamente uno degli uomini meno cattolici che operino oggi nella cultura italiana [...] Forse appunto perché sono così poco cattolico ho potuto amare il Vangelo e farne un film [...] Ho potuto farlo così come l'ho fatto, perché mi sento libero, e non ho paura di scandalizzare nessuno; e, infine, perché sento che la parola d'amore (incapacità di concepire discriminazioni manichee, istinto di gettarsi aldilà delle abitudini, sempre, sfidando ogni contraddizione), parola d'amore di cui è stato campione Giovanni XXIII, va considerata un impegno nella nostra lotta. ${ }^{36}$

Papa Giovanni, con Kennedy e Kruscev, costituirà nei primi anni '60 la principale fonte di speranza di un mondo nuovo, che Pasolini condividerà con milioni di uomini. Sullo stesso periodico (ottobre 1964), si fa notare, peraltro, l'influenza dell'amata filologia nella formazione del "papa buono»:

Non c'è nulla di più follemente aberrante del razzismo. Ora, da parte dei comunisti verso i preti, e da parte dei preti verso i comunisti, c'è una specie di atteggiamento razzistico: essi, volendolo o no, cedono a una specie di tentazione discriminatoria, che svaluta l'interezza umana e storia dell'altro [...] Papa Giovanni era incapace di discriminare, di vedere nell'uomo l'altro, il nemico per definizione [...]. Ho saputo in questi giorni che quando era a Istanbul, egli frequentava le lezioni di filologia e di critica stilistica di Auerbach; e questo mi spiega molte cose, non solo il suo particolare modo di fare "lo spirito" (che è tipico della persona raffinatamente specializzata), ma del «distacco» luminoso che egli aveva dalle cose della vita, dello sguardo globale che egli gettava sul mondo, al di là delle sue folli discriminazioni. ${ }^{37}$

L'articolo si conclude con due affermazioni che diverranno pietre angolari nella storia del dialogo tra marxisti e cristiani in Italia: 1) «Una filosofia atea non è la sola filosofia possibile del marxismo»; 2) «Il grande nemico di Cristo non è il materialismo comunista, ma il materialismo borghese». ${ }^{38}$

35. Ibid., p. 181-183; e cfr. anche p. 76-78 e 136-140.

36. Ibid., p. 222-224.

37. Ibid., p. 225-226.

38. Ibid., p. 226. Pasolini fu senz'altro uno dei più convinti sostenitori di tale dialogo. In merito, rivelatrice la conferenza «Marxismo e cristianesimo» tenuta a Brescia (dicembre 1964), ora SPS, p. 786-824. In uno dei passi centrali si afferma: «nel fondo dell'azione di Marx c'è un profondo spiritualismo» (ibid., p. 801). 


\section{Dalla critica del linguaggio tecnocratico alla critica della televisione}

I testi che ancor più rivelano la creativa assimilazione del pensiero gramsciano, sono quelli scritti da Pasolini tra il 1964 e il 1965, e raccolti poi in Empirismo eretico. Il primo di essi, Nuove questioni linguistiche, esce su Rinascita (26 dicembre 1964), ma il contenuto è anticipato in Vie Nuove, il mese prima, con un esplicito riferimento al sardo:

si ricordi Gramsci: ogni volta che si ripropone la questione della lingua, vuol dire che si ripropongono problemi sociali e politici di fondo, diceva Gramsci, pressappoco [...] spero di avere finalmente centrato l'argomento. ${ }^{39}$

L'avvio del saggio è piuttosto incerto e sfocato, tradendo l'origine orale del testo; l'argomentazione diventa più convincente quando si comincia a spiegare perché «in Italia non esiste una vera e propria lingua italiana nazionale»:

la lingua italiana è la lingua della borghesia italiana che per ragioni storiche determinate non ha saputo identificarsi con la nazione, ma è rimasta classe sociale: [...] è la lingua delle sue abitudini, dei suoi privilegi, delle sue mistificazioni, insomma della sua lotta di classe. ${ }^{40}$

La parte più originale è quella centrale che, analizzando il linguaggio politico e giornalistico, diffuso dai mass-media, vi coglie le spie dei cambiamenti profondi in corso nella società italiana. Pasolini si sofferma, in particolare, su un brano di un discorso di Aldo Moro, teletrasmesso in occasione dell'inaugurazione dell'autostrada del sole. Sembra utile riportare le acute osservazioni anche per la loro straordinaria attualità in tempi di crescente tecnocrazia:

non si tratta di un discorso a tecnici come il quantitativo di terminologia tecnica potrebbe far credere; si tratta di un discorso a un pubblico normale [...] di tutte le condizioni, le culture, i livelli, le regioni [...] le sue frasi crudamente tecniche hanno addirittura una funzione di captatio benevolentiaes sostituiscono quei passi che un tempo sarebbero stati di perorazione e enfasi. Infatti Moro strumentalizza l'inaugurazione dell'autostrada per fare un appello politico agli italiani [...]: quello di cooperare al superamento della congiuntura. [...] Una tale raccomandazione nell'italiano che noi siamo abituati a considerare nazionale, avrebbe richiesto un tour de force dell' ars dictandi [...]. Qualcosa di fondamentale è dunque successo alle radici del linguaggio politico ufficiale. ${ }^{41}$

Per Pasolini la tecnologia dominante nelle società altamente industrializzate ha trasformato anche il linguaggio politico. Mentre, fino a ieri, l'osmosi con il latino, tendeva a differenziarlo dagli altri linguaggi, la tecnologia tende

39. Le belle bandiere, cit., p. 229.

40. Empirismo eretico, cit., p. 5-6.

41. Ibid., p. 17. 
ad omologare tutto. Televisione e giornali sono stati i primi a registrare questo cambiamento epocale per il quale "centri creatori, elaboratori e unificatori di linguaggio, non sono più le università, ma le aziende». ${ }^{42}$

Un ulteriore segno della rivoluzione in corso è dato dal "potere di suggestione linguistica enorme che hanno gli slogan nel linguaggio della pubblicità». Pasolini sa che si tratta di un linguaggio vero e proprio, i cui principi cominciano a passare nello stesso parlato. In esso, scrive, «il principio omologatore e direi creatore è la tecnologia». ${ }^{43}$

Per lui il fenomeno tecnologico investe la lingua, «come una nuova spiritualità», dalle radici a tutte le sue ramificazioni. E ciò perché «è finito un tipo di società $[. .$.$] e ne è cominciato un altro». { }^{44}$

Quando esce questo saggio, non era in corso alcun dibattito sui mutamenti linguistici in atto nella società italiana. Pasolini era rimasto colpito, fin dai primi anni '60, dagli sconvolgimenti sociali che stavano cambiando il volto dell'Italia (crisi della secolare civiltà contadina, urbanesimo, emigrazioni interne ed esterne, imborghesimento della classe operaia, esplosione dei mass-media) e dalle loro ricadute sul terreno culturale e linguistico. Nessun altro, prima di lui, aveva percepito questo terremoto. E sarà forse stato proprio lo sconfinamento nel terreno sociologico, a fare arricciare il naso ai linguisti e ai semiologi del tempo, chiusi nelle loro fortezze e certezze disciplinari.

Insieme all'avvento del neocapitalismo, Pasolini è in Italia tra i primi a segnalare la crisi dei partiti che si richiamano al marxismo. Così proprio su Vie Nuove publicherà nell'estate del 1965 Due crisi. ${ }^{45}$ Egli è convinto che la crisi del marxismo è determinata, da un lato, dallo «sviluppo in qualche modo rivoluzionario del neocapitalismo", dall'altro, dal persistente stalinismo, sopravvissuto a Stalin e agli stessi Kruscev e Togliatti. Anche per questo chiederà al PCI «un'autocritica reale degli errori del passato» ${ }^{46}$ e, pur rimasto senza risposta e costretto a interrompere la collaborazione con Vie nuove, continuerà a cercare dentro il PCI e il suo eterodosso marxismo la via d'uscita dalla crisi.

Pasolini sa che «nessuna formula è mai buona per superare i momenti critici della realtà»e, poiché "ogni cosa si muove col muoversi della storia» e «non esistono cose immobili», si richiama ancora una volta a Gramsci per esortare tutti a "guardare in faccia la realtà così com'è». ${ }^{47}$ Egli è consapevole di avere rotto una lunga tradizione: da Gramsci ha appreso che "gli intellettuali

42. Ibid., p. 18. Qualche anno dopo scriverà (Il caos, Roma: Editori Riuniti, 1979, p. 41): "L'egemonia culturale, che per circa un ventennio è stata del PCI, è passata nelle mani dell'industria".

43. Il passo anticipa di quasi due lustri l'analisi linguistica della réclame dei jeans Jesus in Scritti corsari.

44. Ibid., p. 22.

45. Le belle bandiere, cit., p. 315-316.

46. Ibid., p. 324-325.

47. Empirismo eretico, cit., p. 46. 
italiani sono sempre stati cortigiani» e sono sempre vissuti «dentro il Palazzo», ${ }^{48}$ perciò li considera corresponsabili dell' «orrendo potere» costruitosi in Italia dalla prima metà degli anni ' 60 . La critica contro questo potere, come visto, comincia allora, ma diventa più serrata, sfiorando talora l'invettiva, negli ultimi anni della sua vita. Per rendersene conto, basta rileggere gli articoli scritti tra il 1973 e pochi giorni prima della tragica morte:

I potenti democristiani che in questi anni hanno detenuto il potere, dovrebbero andarsene, sparire, per non dire di peggio. Invece non solo restano al potere, ma parlano. Ora è la loro lingua che è la pietra dello scandalo. Infatti ogni volta che aprono bocca, essi, per insincerità, per colpevolezza, per paura, per furberia, non fanno altro che mentire. La loro lingua è la lingua della menzogna. E poiché la loro cultura è una putrefatta cultura forense e accademica, mostruosamente mescolata con la cultura tecnologica, in concreto la loro lingua è pura teratologia. Non la si può ascoltare, bisogna tapparsi le orecchie. Il primo dovere degli intellettuali, oggi, sarebbe quello di insegnare alla gente a non ascoltare le mostruosità linguistiche dei potenti democristiani, a urlare, a ogni loro parola, di ribrezzo e di condanna. In altre parole, il dovere degli intellettuali sarebbe quello di rintuzzare tutte le menzogne che attraverso la stampa e soprattutto la televisione inondano e soffocano quel corpo del resto inerte che è l'Italia. ${ }^{49}$

Questo testo del 25 marzo 1975 può considerarsi il manifesto programmatico del Pasolini corsaro e luterano, e aiuta a capire alcuni dei motivi per cui prese a collaborare contraddittoriamente con due giornali borghesi per continuare la battaglia avviata quindici anni prima su Vie Nuove.

Soprattutto mirato pare l'articolo Sfida ai dirigenti della televisione (Corriere della sera, 9 dicembre 1973), ${ }^{50}$ che indica la TV quale principale veicolo di quella che, l'anno dopo, sarà definita la mutazione antropologica degli italiani. Per Pasolini neppure il fascismo è riuscito a fare ciò che ha fatto il neocapitalismo con la televisione: il fascismo proponeva un modello, reazionario e monumentale, che però restava lettera morta. Le varie culture particolari (contadine, sottoproletarie, operaie) continuavano imperturbabili a uniformarsi ai loro antichi modelli: la repressione si limitava a ottenere un'adesione a parole. Oggi, invece, l'adesione ai modelli imposti dal Centro è totale e incondizionata a causa del potere della televisione, già entrata in quegli anni in tutte le case:

La responsabilità della televisione [...] è enorme. Non certo in quanto «mezzo tecnico", ma in quanto strumento del potere e potere essa stessa. [...]. Ė attraverso lo spirito della televisione che si manifesta in concreto lo spirito del nuovo potere. [...] Il fascismo, voglio ripeterlo, non è stato in grado nemmeno di scalfire l'anima del popolo italiano: il nuovo fascismo, attraverso i nuovi mezzi

48. Lettere luterane, cit., p. 93-94.

49. Ibid., p. 29.

50. Senza la parte conclusiva, in cui si articolava la sfida, finisce in Scritti corsari come Acculturazione e acculturazione (sic), quasi a sottolineare il prevalente taglio antropologico. 
di comunicazione e di informazione (specie, appunto, la televisione), non solo l'ha scalfita, ma l'ha lacerata, violata, bruttata per sempre. ${ }^{51}$

Va ricordato che Pasolini aveva colto, fin dal 1958, in un'intervista dal titolo premonitore, la «funzione livellatrice» della TV, la sua potente capacità di diffondere, anche indirettamente, "una visione unitaria della vita e del mondo". 52

Nel 1963, conversando con Arbasino, affermerà con straordinaria preveggenza:

Si produrrà e si consumerà, ecco. E il mondo sarà esattamente come oggi la televisione - questa degenerazione dei sensi umani- ce lo descrive con stupenda, atroce ispirazione profetica. ${ }^{53}$

Nello stesso anno arriva nei cinema La rabbia..$^{54}$ In una scena lo speaker del cinegiornale annuncia trionfante che presto gli abbonati della TV saranno «decine di migliaia»; Pasolini lo corregge: «No. Saranno milioni. Milioni di candidati alla morte dell'anima. Il nuovo mezzo è stato inventato per la diffusione della menzogna». 55

La televisione occupa un posto centrale anche nei saggi linguistici del biennio 1964-65, prima esaminati, in cui le è attribuito anche il merito di avere operato la prima unificazione linguistica nazionale.

Come si vede, dietro ai giudizi trancianti degli ultimi anni, c'è più di un decennio di riflessioni. Così, chi aveva scoperto «il linguaggio delle cose», negli ultimi mesi di vita sperimenta l'impotenza delle sue denunce e della sua pedagogia di fronte alla prepotenza della TV che:

altro non fa che offrire una serie di «esempi» [...]. Anche se annunciatori, presentatori parlano (e orrendamente) in effetti il vero linguaggio della televisione è simile al linguaggio delle cose: è perfettamente pragmatico e non ammette repliche, alternative, resistenze. ${ }^{56}$

Tuttavia, pochi giorni prima di essere trucidato, Pasolini precisa pubblicamente che tanti equivoci sul suo pensiero sono sorti per il suo stile comunicativo provocatorio e paradossale, e smentisce la visione apocalittica data di sé in qualche momento. ${ }^{7}$

51. Ibid., p. 30.

52. "Neocapitalismo televisivo». Intervista a cura di Arturo GISMONDI, Vie Nuove, XIII 50, 20 dicembre 1958, ora SPS, p. 1553-1559.

53. SPS, p. 1572.

54. Il film, nella sua versione originale, è stato restaurato nel 2008 da Giuseppe Bertolucci.

55. Curzio MAltese, «Il film-profezia di Pasolini, così nel '63 raccontò l'Italia d'oggi», La Repubblica, 28 agosto 2008.

56. Lettere luterane, cit., p. 36-37.

57. Volgar'eloquio, cit., ora SLA, II, p. 2846: «non ho nulla contro la scuola e la televisione come strumenti. Sono strumenti meravigliosi, la televisione soprattutto. Pensa un po'cosa potrebbe essere la televisione». 
Il tema specifico della mutazione antropologica degli italiani sarà affrontato nell'articolo Gli italiani non sono più quelli, divenuto poi il «corsaro» Studio sulla rivoluzione antropologica in Italia. Prendendo spunto dall'esito del referendum sul divorzio, scandalizzando molti suoi amici, Pasolini sostiene che la vittoria del NO è una sconfitta non solo di Fanfani e del Vaticano, ma anche di Berlinguer e del partito comunista, a cui è ancora vicino. ${ }^{58} \mathrm{Il}$ rimprovero è di non aver capito nulla delle trasformazioni profonde avvenute in Italia negli ultimi dieci anni. ${ }^{59}$

L'articolo suscita un vespaio di polemiche. Pasolini è accusato di estetismo e irrazionalismo, di mancanza di senso storico: Calvino gli rimprovera di rimpiangere l'Italietta provinciale e contadina ${ }^{60}$ ed altri gli ricorderanno l'elogio della civiltà borghese contenuto nel Manifesto dei comunisti del 1848. Ma il corsaro replica a tutti:

È stata la propaganda televisiva del nuovo tipo di vita edonistico che ha determinato il trionfo del $\mathrm{NO}$ al referendum. [...] E vero che in tutti questi anni la censura televisiva è stata una censura vaticana. Solo però che il Vaticano non ha capito che cosa doveva censurare. Doveva censurare per esempio "Carosello", perché è in Carosello, onnipotente, che esplode in tutto il suo nitore, la sua assolutezza, il nuovo tipo di vita che gli italiani «devono» vivere [...] Il bombardamento ideologico televisivo non è esplicito: esso è tutto nelle cose, tutto indiretto. ${ }^{61}$

Ad altri -e a Calvino soprattutto, dal cui intervento si sentì profondamente ferito ed offeso- risponderà con una lettera aperta su Paese Sera (8 luglio 1974):

Che degli altri abbiano fatto finta di non capire è naturale. Ma mi meraviglio che non abbia voluto capire tu (che non hai ragioni per farlo). Io rimpiangere l'«Italietta»? Ma allora tu non hai letto un solo verso delle Ceneri di Gramsci o di Calderón, non hai letto una sola riga dei miei romanzi, non hai visto una sola inquadratura dei miei films, non sai niente di me! Perché tutto ciò che io ho fatto e sono, esclude per sua natura che io possa rimpiangere l'Ita-

58. Cfr. Che cos'è questo golpe?, dove arriva a definire il PCI «un paese pulito in un paese sporco, un paese onesto in un paese disonesto, un paese intelligente in un paese idiota». Ora Scritti corsari, cit., p. 110.

59. Ibid., p. 47-48.

60. Calvino interviene nel dibattito dalle colonne del Messaggero (18 giugno 1974). Ma non è l'unico a farlo. Cfr. i pareri di Alberto Moravia, Lucio Colletti, Franco Fortini e Leonardo Sciascia, pubblicati da L'Espresso la settimana dopo l'articolo di Pasolini. Non a caso sarà proprio Sciascia a mostrarsi il più comprensivo e generoso nei suoi confronti. A distanza di anni, la critica è ancora divisa sulla valutazione del Pasolini corsaro. Accanto ai Sanguineti e Guglielmi che hanno, da sempre, giudicato reazionaria l'analisi pasoliniana, ci sono altri (Betti, Mantegazza) che la reputano rivoluzionaria. Una posizione più equilibrata pare quella di Alfonso Berardinelli, Prefazione a P. P. Pasolini, Scritti corsari, Nuova ed., Milano: Garzanti, 2007, p. VII-X.

61. Ibid., p. 69-70. 
lietta. A meno che tu non mi consideri radicalmente cambiato: cosa che fa parte della psicologia miracolistica degli italiani, ma che appunto per questo non mi par degna di te. ${ }^{62}$

Qui Pasolini, oltre a rivendicare la continuità di fondo della sua opera, fa un primo tentativo di precisare il senso del suo rimpianto della «millenaria civiltà contadina»: ${ }^{63}$

L'universo contadino (cui appartengono le culture sottoproletarie urbane, e, appunto fino a pochi anni fa, quelle delle minoranze operaie [...]) è un universo transnazionale [...]. È questo illimitato mondo contadino prenazionale e preindustriale, sopravvissuto fino a pochi anni fa, che io rimpiango (non per nulla dimoro il più a lungo possibile, nei paesi del Terzo Mondo, dove esso sopravvive ancora, benché il Terzo Mondo stia anch'esso entrando nell'orbita del cosiddetto Sviluppo). ${ }^{64}$

Per non fraintendere lo scrittore, certo consapevole che gli uomini in carne ed ossa di questo mitico "universo contadino» non vivevano in un' età dell'oro, occorre tenere sempre presente che il suo punto di vista non è quello del sociologo, che guarda con distacco le cose, ma quello del poeta che ama e soffre osservando, con tutti i sensi e non solo con la ragione, i cambiamenti in corso. ${ }^{65}$ Ciò si manifesta in particolare nei testi in prosa scritti nel biennio 1973-74 insieme ai nuovi versi friulani di La nuova gioventù, in cui il poeta polemizza con il riformismo del PCI del tempo, ritenuto subalterno al «modello di sviluppo» capitalistico:

Il «modello di sviluppo» è quello voluto dalla società capitalistica che sta per giungere alla massima maturità. Proporre altri modelli di sviluppo, significa accettare tale primo modello di sviluppo. [...] No: non bisogna accettare tale «modello di sviluppo». [...] Bisogna rifiutare lo «sviluppo». Questo «sviluppo»: perché è uno sviluppo capitalista [...]. I comunisti che accettano questo «sviluppo", considerando il fatto che l'industrializzazione [...] è irreversibile, sarebbero indubbiamente realisti a collaborarvi, se la diagnosi fosse assolutamente giusta e sicura. E invece non è detto [...] che tale «sviluppo» debba continuare com'è cominciato. C'è anzi la possibilità di una «recessione». ${ }^{66}$ Cinque anni di «sviluppo» hanno reso gli italiani un popolo di nevrotici idioti, cinque anni di miseria possono ricondurli alla loro sia pur misera umanità. ${ }^{67}$

62. Ibid., p. 60. Corsivo d'A. La lettera (ibid., p. 60-65) sarà titolata Limitatezza della storia e immensità del mondo contadino. Calvino e Pasolini torneranno a confrontarsi nell'ottobre 1975. Per quest'ultimo scambio epistolare, cfr. Lettere Luterane, cit., p. 179-184.

63. Scritti corsari, cit., p. 32.

64. Ibid., p. 62.

65. Giovanni Raboni non ha tutti i torti, quando paradossalmente afferma che c'è più poesia nella prosa e nel cinema di Pasolini che nei suoi versi. Cfr. P. CONTI, «Raboni: poeta senza poesia", Corriere della sera, 2 novembre 1995.

66. Si ricordi che nel 1973 l'occidente aveva conosciuto la prima crisi petrolifera mondiale, a seguito della rivoluzione in Iran.

67. La nuova gioventù, cit., p. 241. Corsivo d'A. 
L'originalità dell'analisi non va però ricercata in tale critica. I fogli extraparlamentari del tempo — Lotta Continua in testa - erano pieni di attacchi simili alla subalternità del PCI. L'originalità sta tutta nell'attacco allo «sviluppo» e nell'affermazione eretica — dal punto di vista marxista — secondo cui lo sviluppo economico e l'industrializzazione, di per sé, non sono portatori di Progresso.

Per quanti dubbi possano nutrirsi sulla razionalità dell'analisi, non è esagerato intravedervi un'anticipazione della teoria della «decrescita» avanzata dall'economista Serge Latouche. ${ }^{68}$ Pasolini aveva l'onestà di riconoscere la difficoltà di argomentare in modo scientifico il suo pensiero. Anche per questo, forse, riprende a scrivere versi in friulano proprio in quegli anni: ${ }^{69}$

si torna indietro? Stupida verità.

Jo mi vuàrdi indavour, e i plans

i paìs puòrs $[\ldots]$

I plans un mond muàrt.

Ma i no soj muàrt jo ch'i lu plans.

Si vulin zi avant bisogna ch'i planzini

il timp ch'a no'l pòs pi tornà, ch'i dizini di no

a chista realtàt ch'a ni à sieràt

ta la so preson... ${ }^{70}$

Il testo friulano a questo punto s'interrompe per proseguire in italiano. Non credo per una presunta "aridità creativa» dell'autore, quanto per il permanente carattere pedagogico della sua scrittura. ${ }^{71}$ Così i versi incalzano:

L'hanno costruita i signori: cioè

i nemici di classe. Adesso hanno delle difficoltà.

Noi dovremmo dargli una mano? Certo se fosse

loro il futuro, ciò sarebbe realistico...

Ma non saremo stati troppo lucidi

(la lucidità del piacere di morire)

a credere che quella loro realtà

fosse quella di tutto il futuro? ${ }^{72}$

68. Cfr. almeno S. Latouche, Petit traité de la décroissance sereine, Paris: Fayard, 2007.

69. Cfr. Duflot, cit., p. 5. In un'altra pagina autobiografica - Scritti corsari, cit., p. 221222 - commenta magistralmente alcuni versi di Ignazio Buttitta e spiega le ragioni che l'hanno spinto a riscrivere in friulano.

70. Ibid., p. 236-237. A piè di pagina, Pasolini offre la versione italiana: «Io mi guardo indietro, e piango i paesi poveri [...] Piango un mondo morto. Ma non sono morto io che lo piango. Se vogliamo andare avanti, bisogna che piangiamo il tempo che non può più tornare, che diciamo di no a questa realtà che ci ha chiusi nella sua prigione...".

71. È lui stesso (Lettere luterane, cit., p. 15-67) a confessare tale tratto prevalente. Quanto forte sia stata la sua vocazione pedagogica è stato riconosciuto da tutti e, in particolare, da Enzo Golino, Pasolini. Il sogno di una cosa. Pedagogia, Eros, Letteratura dal mito del popolo alla società di massa, Milano: Bompiani, 1992.

72. La nuova gioventù, cit., p. 237. Un po'piú giú (p. 245), l'Appunto per una poesia in terrone, radicalizza ulteriormente il suo punto di vista: «Così non si può andare avanti. Perché avete lasciato che i nostri figli fossero educati dai borghesi? Perché avete permesso che le nostre 
Pasolini aveva indubbiamente ragione di considerare questi testi più corsari di quelli noti come tali. ${ }^{73}$ In essi, infatti, si anticipano alcuni dei temi del famoso articolo Il vuoto di potere in Italia (Corriere della sera, 1 febbraio 1975):

Gli uomini di potere democristiano sono passati dalla «fase delle lucciole» alla fase della «scomparsa delle lucciole» senza accorgersene [...] non hanno sospettato minimamente che il potere che essi detenevano e gestivano, non stava semplicemente subendo una normale evoluzione, ma stava cambiando radicalmente natura [...] Gli uomini di potere DC hanno subito tutto questo, credendo di amministrarlo. Non si sono accorti che esso era "altro»: incommensurabile non solo a loro ma a tutta una forma di civiltà. Come sempre (cfr. Gramsci) solo nella lingua si sono avuti dei sintomi. Nella fase di transizione [...] gli uomini di potere DC hanno bruscamente cambiato il loro modo di esprimersi, adottando un linguaggio completamente nuovo (del resto incomprensibile come il latino): specialmente Aldo Moro: cioè (per una enigmatica correlazione) colui che appare il meno implicato di tutti nelle cose orribili che sono state organizzate dal ' 69 a oggi, nel tentativo, finora formalmente riuscito, di conservare comunque il potere. ${ }^{74}$

La densità del brano richiederebbe un commento particolareggiato. Per ora rilevo solo che non è casuale che Sciascia, ${ }^{75}$ tra i pochi ad averlo compreso

case fossero costruite dai borghesi? Perché avete tollerato che le nostre anime fossero tentate dai borghesi? Perché avete protestato solo a parole mentre pian piano la nostra cultura (sapere, modo di essere) si andava trasformando in una cultura borghese?».

73. Da buon filologo quale era, egli conosceva il significato delle parole e, come pochi, sapeva usarle. Se decise d'intitolare l'ultimo libro Scritti corsari, una ragione doveva esserci. Il termine corsaro, si sa, è equivoco o, se si preferisce, polisemico. I dizionari storici distinguono i corsari dai pirati, perché i primi, a differenza dei secondi, erano autorizzati ad issare una speciale bandiera e a praticare in mare la cosiddetta guerra di corsa. Nel senso comune odierno tale differenza è andata smarrita. Credo però che, in questo caso, la filologia aiuti più della storia ad avvicinarci al significato nascosto attribuito da Pasolini a corsaro; essa, infatti, rivela l'etimo mediolatino: cursariu $(m)$, deriv. da cursus 'viaggio per mare' e da curre$r e$ 'correre'. Ricordando, peraltro, il costante impegno di Pasolini a «non adattare l'orizzonte al periscopio, ma il periscopio all'orizzonte, all'immenso orizzonte dei fenomeni», vedo in corsaro il senso di "corsa in mare aperto». D'altronde, se ci si pensa bene, cos'altro è stata la vita di Pasolini, prima ancora della sua opera?

74. Scritti corsari, cit., p. 160-163.

75. È singolare che, pur essendo tanto diversi per formazione e temperamento, i due si siano ritrovati, alla fine, sulla stessa lunghezza d'onda. Assai toccante la testimonianza resa da Sciascia dopo la morte di Pasolini: «io mi sentivo sempre un suo amico; e credo anche lui nei miei riguardi. C'era però come un ombra tra noi, ed era l'ombra di un malinteso. Credo che mi ritenesse alquanto — come dire? — razzista nei riguardi dell'omosessualità. E forse era vero, e forse è vero: ma non al punto da non stare dalla parte di Gide contro Claudel, dalla parte di Pier Paolo Pasolini contro gli ipocriti, i corrotti e i cretini che gliene facevano accusa. E il fatto di non essere mai riuscito a dirglielo mi è ora di pena, di rimorso. Io ero — e lo dico senza vantarmene, dolorosamente - la sola persona in Italia con cui lui potesse veramente parlare. Negli ultimi anni abbiamo pensato le stesse cose, dette le stesse cose, sofferto e pagato per le stesse cose. Eppure non siamo riusciti a parlarci, a dialogare». (Nero su nero, Torino: Einaudi, 1979, p. 175-176). Sciascia curò il prezioso volumetto con versi 
fino in fondo, apra L'affaire Moro richiamandosi proprio ad esso. In secondo luogo, noto che l'esplicito rimando a Gramsci conferma la persistente e decisiva influenza del sardo nell'ultimo Pasolini.

Il cerchio attorno ai dirigenti democristiani si stringe ancor di piú nell'articolo Il Processo (Corriere della sera, 24 agosto 1975). Colpisce, ancor oggi, l'apertura in cui si elencano i reati contestati alla classe politica del tempo:

Disprezzo per i cittadini, manipolazione di denaro pubblico, intrallazzo con i petrolieri, con gli industriali, con i banchieri, connivenza con la mafia, alto tradimento in favore di una nazione straniera, uso illecito dei servizi segreti, responsabilità nelle stragi di Milano, Brescia e Bologna [...], distruzione paesaggistica e urbanistica dell'Italia. ${ }^{76}$

Bisognerà aspettare quasi vent'anni per sentire l'eco della solitaria denuncia pasoliniana in aule giudiziarie. Gli unici politici contemporanei che, pur in ritardo, non rimasero insensibili ad essa si chiamavano Enrico Berlinguer e Ugo La Malfa. ${ }^{77}$ Non a caso, i due incontrarono più di una resistenza all'interno dei loro stessi partiti, ma ciò non avrebbe sorpreso chi aveva scritto che «il coraggio intellettuale della verità e la pratica della politica sono due cose inconciliabili in Italia». ${ }^{78}$

La spietata analisi del consumismo e del conformismo dilaganti voleva essere soprattutto un grido d'allarme contro i pericoli incombenti intravisti all'orizzonte e indicati chiaramente nell'ultima profetica intervista:

State attenti. L'inferno sta salendo da voi.[...] Non vi illudete. Voi siete, con la scuola, la televisione, la pacatezza dei vostri giornali, voi siete i grandi conservatori di questo ordine orrendo basato sull'idea di possedere. ${ }^{79^{9}}$

Nella trascrizione che Pasolini non fece in tempo a rivedere, si trova una delle migliori definizioni del concetto di potere da lui formulate:

Il potere è un sistema di educazione che ci divide in soggiogati e soggiogatori. [...] Uno stesso sistema educativo che ci forma tutti, dalle cosiddette classi dirigenti, giù fino ai poveri. Ecco perché tutti vogliono le stesse cose e si com-

giovanili: P. P. PASOlInI, Dal diario (1945-47), Caltanissetta: Salvatore Sciascia, 1979, chiosato cosí nell'introduzione: «dopo aver riletto queste sue poesie, mi pare di aver vissuto una lunghissima vita e che la felicità di allora sia come il ricordo di un altro me stesso; un lontano e remoto me stesso, non il me stesso di ora. Eravamo davvero così giovani, così poveri, così felici?».

76. Lettere luterane, cit., p. 114-123.

77. È nota la levata di scudi contro Berlinguer, quando propose la politica dell'austerità e la centralità della questione morale. Meno nota, invece, l'attenzione prestata da La Malfa al poeta, venuta alla luce in un suo inedito (Corriere della sera, 6 maggio 2004).

78. Scritti corsari, cit., p. 110.

79. P. P. PASOlInI, «Siamo tutti in pericolo», Intervista a cura di Furio ColOMbO, La Stampa, Tuttolibri, 18 novembre 1975, ora SPS, p. 1728-1729. 
portano nello stesso modo [...] L'educazione ricevuta è stata: avere, possedere, distruggere. ${ }^{80}$

\section{Nostalgia del volgar'eloquio e bisogno di un nuovo modo d'essere gramsciani}

Ormai in conclusione, non posso eludere l'ultimo intervento pubblico in cui Pasolini affronta di petto il tema suggerito dal titolo di questo saggio.

E un documento di fondamentale importanza, colpevolmente trascurato dalla critica; ${ }^{81}$ una sorta di summa, in cui si ritrovano i principali temi dibattuti appassionatamente dall'autore nel corso della breve vita. Esso, peraltro, conferma la sostanziale coerenza della riflessione di Pasolini e la centralità che ha nella sua opera la questione dei rapporti tra lingua e potere.

L'intervento, come accennato, si svolse al Liceo Palmieri di Lecce il 21 ottobre 1975, pochi giorni prima della tragica morte, nell'ambito di un Corso di aggiornamento per docenti di Scuola Media Superiore sul tema «Dialetto a scuola».

Fu Pasolini a voler dare all'incontro il titolo dantesco, così evocativo della tradizione letteraria italiana. ${ }^{82}$ Lo scrittore esordisce affermando di non saper parlare e di non essere in grado di tenere una lezione. Propone, pertanto, di passare immediatamente al dibattito. Ma di fronte al silenzio imbarazzato degli astanti decide di leggere, come introduzione, il monologo finale del dramma, allora inedito, Bestia da stile, che gli ha fornito l'idea d'intitolare l'incontro in quel modo bizzarro.

Ecco solo i versi iniziali della nota poesia, per rendersi conto del suo stile comunicativo e del singolare rapporto con le patrie lettere: ${ }^{83}$

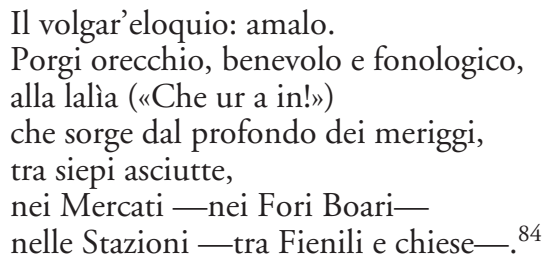

80. Ibid., p. 1725-1726.

81. Si rimanda alla bibliografia discussa in apertura.

82. La prima trascrizione, come ricordato, si deve a A. PIrOMAlli, Volgar'eloquio, cit.

83. Una delle contraddizioni più feconde e persistenti nell'opera di Pasolini è l'uso democratico dell'aristocratica tradizione letteraria nazionale. Su di essa si sono soffermati diversi critici. Per tutti, cfr. Alberto Moravia, "Pasolini poeta civile» (1977), in AA.VV., Per conoscere Pasolini, cit., p. 7-10 e Arcangelo LeOne De CAstris, Sulle ceneri di Gramsci. Pasolini, i comunisti e il 68, Napoli: CUEN, 1993, p. 31-32.

84. SLA, p. 2825-2827. 
Era uno spunto dichiaratamente provocatorio. Come confermano vari testimoni, lo smarrimento dell'uditorio, a lettura finita, non poteva che crescere, tanto più che l'invito ad amare il Volgar'eloquio era rivolto a un inesistente giovane di un'immaginaria "Destra sublime», che solo Pasolini poteva inventarsi!

Ma per comprendere meglio quanto avvenne quel giorno, conviene riprendere le parole di uno degli organizzatori dell'incontro, Gustavo Buratti, presidente dell'AIDLCM (Associazione internazionale per la difesa delle lingue e delle culture minacciate), che aveva direttamente invitato Pasolini, ricordandogli l'antica «militanza» per quelle che Frédéric Mistral chiamava «li lengo meprisado» (le lingue disprezzate):

Ma questi temi (l'amore per il «volgar'eloquio» e l'impegno conseguente), diceva Pasolini, sono una specie di palla al piede per noi, uomini della sinistra. [...] Tuttavia, noi che abbiamo lottato per la nostra lingua, sappiamo quanto Pasolini avesse ragione... sovente i nostri discorsi sono travisati; siamo accusati di dividere, con problematiche sovrastrutture, la classe operaia; di «fare il gioco dei padroni» e della destra, magari financo di essere razzisti. ${ }^{85}$

In effetti, nell'intervento, Pasolini ammette di non avere alcuna ricetta:

È tutto problematico, ed è problematica soprattutto un'azione politica chiara, che non vedo da nessuna parte. ${ }^{86}$

Torna allora a discutere dei rapporti tra lingua e dialetti, con cui aveva fatto i conti fin da giovane; rimette a fuoco, aggiornando l'analisi, l'annosa questione dei rapporti tra lingua e società, evidenzia come i rapidi mutamenti delle abitudini linguistiche degli italiani fossero uno dei frutti della scomparsa della civiltà contadina. Nel riconoscere infine la crisi della vecchia ideologia marxista-leninista, incapace di comprendere il neocapitalismo, invoca la necessità di «un nuovo modo d'essere gramsciani». Più precisamente, dopo aver rivendicato con orgoglio di essere stato un marxista critico da trent'anni ${ }^{87}$ e di aver dato un contributo originale allo storicismo gramsciano, afferma, memore della classica lezione marxiana:

bisogna tenere presente l'assioma primo e fondamentale dell'economia politica, cioè che chi produce non produce solo merci, produce rapporti sociali, cioè umanità. 88

Ora, aggiunge Pasolini, dato che il neocapitalismo ha rivoluzionato il vecchio modo di produzione e tramite la produzione di beni superflui e il consu-

85. G. BurATTI, «Pasolini: dialetto rivoluzionario e minoranze linguistiche», cit.

86. SLA, p. 2854.

87. Ibid., p. 2839.

88. Ibid., p. 2840. Il passo è, di fatto, una delle più ortodosse espressioni della critica marxiana al sistema di produzione capitalistico. 
mismo ha trasformato antropologicamente gli italiani, i vecchi comunisti non sanno più cosa fare. Nella confusione tendono a trasformarsi in «un nuovo tipo di chierici» che, non tenendo conto dei cambiamenti profondi avvenuti negli ultimi dieci anni, ripetono salmodicamente il catechismo marxista-leninista, accusando di eresia tutti coloro che la pensano diversamente:

dove ho scritto che bisogna ritornare indietro? Dove? Vedete punto per punto, e io [...] vi dico no: avete capito male, vi siete sbagliati, non intendo affatto ritornare indietro, appunto perché mi pongo i problemi più attuali, fiuto i problemi del momento [...] Gramsci lavorava quaranta anni fa, in un mondo arcaico che noi non osiamo neppure immaginare [...] puoi ricordarmi Gramsci come anello di una catena storica che porta a fare nuovi ragionamenti oggi, a riproporre un nuovo modo di essere progressisti, un nuovo modo di essere gramsciani. ${ }^{89}$

Come si vede, anche queste parole confermano l'immagine data di sé nell'intervista ad Arbasino del 1963: «la mia caratteristica principe è la fedeltà».

Rileggendo gli scritti di Pasolini si rimane colpiti dalla loro intatta forza espressiva e comunicativa, dalla loro resistenza al tempo. Il fatto stesso che alcune sue parole-chiave (Palazzo, omologazione, mutazione antropologica, sviluppo senza progresso) siano diventate senso comune mi sembra un'ulteriore prova dell'attualità della sua analisi. Soprattutto centrata è la critica al consumismo, percepito e vissuto come "un vero e proprio cataclisma antropologico». ${ }^{90}$ È vero che in essa si ritrovano motivi già presenti nella Scuola di Francoforte, ${ }^{91}$ ma sono certo una novità il linguaggio usato, l'estrema chiarezza ed immediatezza che l'hanno resa comprensibile a tutti. Pasolini, con il suo acume antropologico, è stato tra i primi a capire la centralità dei mass media nella società contemporanea. Fin dagli anni ' $60,{ }^{92}$ sviluppando la geniale intuizione gramsciana rilevante lo stretto nesso tra lingua, società e potere, aveva colto nelle prime manifestazioni del linguaggio tecnocratico l'emergere di una nuova classe sociale tendenzialmente egemone. Ma, a differenza di tanti intellettuali odierni, non ebbe paura di andare contro corrente, di mettersi in gioco in prima persona, rompendo schemi e logiche di schieramento consolidati.

Più volte, dopo la sua morte, si è cercato di metterci una pietra sopra. I più cinici hanno persino usato la sua orribile fine per farlo. Solo Sciascia, a modo suo, ha tentato di mantenere viva la sua lezione. E non è un caso che proprio un discepolo di quest'ultimo, Vincenzo Consolo, insieme a pochi altri, abbia utilizzato, in un manifesto del giugno 2000, il lessico di Pasolini per tentare di aggiornarne l'analisi:

89. Ibid., p. 2843-2844.

90. Scritti corsari, cit., p. 135.

91. Penso, in specie, a Minima moralia di T.W. Adorno e a L'uomo a una dimensione di H. MARCUSE. Su questo punto, cfr. un mio precedente saggio: F. VIRGA, «Pasolini corsaro», Colapesce, almanacco di scrittura mediterranea, X.11, 2004, p. 101.

92. Cfr. soprattutto i saggi linguistici di Empirismo eretico, cit. 
Caduto il regime democristiano per corruzione interna, per mafia, per crimini, è subentrato ad esso un partito di destra il cui leader (Silvio Berlusconi) è proprietario (caso unico in Europa) di tre reti televisive, oltre che di giornali e case editrici. Queste reti televisive, che poggiano la loro esistenza e la loro potenza sui messaggi pubblicitari, hanno negli anni inciso enormemente sulla cultura e sulla lingua italiana. La televisione statale, per ragione di concorrenza o di volontaria omologazione, si è conformata alla cifra culturale e stilistica di quella privata. Sempre più piccolo borghese, consumistico, fascista, il paese, telestupefatto, ha perso ogni memoria di sé, della sua storia, della sua identità. L'italiano è diventato un'orrenda lingua, un balbettio invaso dai linguaggi mediatici che non esprime altro che merce e consumo. ${ }^{93}$

$\mathrm{E}$, in un momento in cui l'Italia sembra davvero andare alla deriva, può essere di conforto ricordare che un giovane scrittore come Roberto Saviano, che ha riscosso con il suo Gomorra un meritato successo internazionale, abbia additato tra i suoi maestri il poeta di Casarsa. ${ }^{94}$

93. Per il testo integrale del Manifesto, cfr. Corriere della Sera, 6 giugno 2000.

94. R. SaVIanO, «Io so e ho le prove», Nuovi Argomenti, 32 (2005). 